\title{
CROMATOGRAFIA GASOSA ULTRARRÁPIDA: UMA VISÃO GERAL SOBRE PARÂMETROS, INSTRUMENTAÇÃO E APLICAÇÕES*
}

\author{
Rodrigo Sequinel, Rafael Rodrigues Hatanaka, Carlos Eduardo Gualtieri, Danilo Luiz Flumignan e José Eduardo de Oliveira* \\ Centro de Monitoramento e Pesquisa da Qualidade de Combustíveis, Biocombustíveis, Petróleo e Derivados, Instituto de Química, \\ Universidade Estadual Paulista, Araraquara - SP / Departamento de Química Orgânica, Instituto de Química, Universidade Estadual \\ Paulista, CP 355, 14801-970 Araraquara - SP, Brasil \\ Juliano Passaretti Filho \\ Departamento de Química Analítica, Instituto de Química, Universidade Estadual Paulista, CP 355, 14801-970 Araraquara - SP, Brasil
}

Recebido em 30/6/10; aceito em 21/9/10; publicado na web em 27/10/10

\begin{abstract}
ULTRA FAST GAS CHROMATOGRAPHY: AN OVERVIEW OF THE MAIN PARAMETERS, INSTRUMENTATION AND APPLICATIONS. Since the introduction of GC there has been an ongoing interest in reducing time of analysis resulting in new terms and definitions such as ultra fast gas chromatography (UF-GC). One of the most used definitions describes UF-GC as a technique that combines the employment of short narrow bore column with very fast temperature programming rates producing chromatographic peaks in the range of $50 \mathrm{~ms}$ and allowing separations times in 1-2 min or less. This paper summarizes the analytical approaches, the main parameters involved and the instrumentation towards UF-GC.
\end{abstract}

Keywords: ultra fast gas chromatography; resistive heating; high sampling frequency detectors.

\section{BREVE HISTÓRICO DA REDUÇÃO DO TEMPO DE ANÁLISE EM CROMATOGRAFIA GASOSA}

As primeiras teorias e alguns dos princípios básicos relacionados à redução do tempo de análise em cromatografia gasosa (CG) foram estabelecidos por volta de 1960, quando Scott em 1958 utilizou colunas empacotadas longas com diâmetro interno (d.i.) reduzido capazes de atingir desempenho de 10 pratos efetivos por segundo em uma separação cromatográfica, o que constituiu um grande avanço para a época. ${ }^{1}$ Outro exemplo clássico, foram os estudos de Jonker et al., que conseguiram separações ultrarrápidas de quatro alcanos lineares $(\mathrm{C} 1 \mathrm{a} \mathrm{C} 4)$ em $150 \mathrm{~ms}$ com a utilização de colunas do tipo empacotadas LiChrosorb Si-60, com $32 \mathrm{~mm}$ de comprimento. ${ }^{2}$ Inicialmente ambos os trabalhos não tiveram uma grande repercussão, sendo que no início da década de 80 outros trabalhos ainda viriam a ser publicados relatando as tentativas de aumentar a velocidade de separações cromatográficas, com a utilização de colunas empacotadas. ${ }^{1,3}$

Um dos motivos para esta falta de reconhecimento estava no fato de que as colunas empacotadas utilizadas, algumas das quais preenchidas com partículas de diâmetro relativamente grande, exigiam condições drásticas de pressão para obtenção de separações mais rápidas, por vezes resultando em prejuízo na eficiência da separação. ${ }^{4,5}$ Além disso, o principal objetivo em uma análise cromatográfica era, na época, obter a melhor resolução para diferentes substâncias em uma mistura ou mesmo separar um par crítico de componentes em uma amostra complexa.

A introdução das colunas capilares por Golay, em 1957, veio a suprimir algumas das limitações relacionadas à utilização das colunas empacotadas em separações cromatográficas complexas e foi o grande passo em direção à obtenção de cromatogramas com

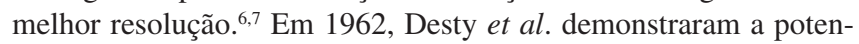
cialidade de colunas capilares com d.i. reduzido para realização de separações rápidas. ${ }^{8}$ Outro trabalho relevante foi o de Gaspar et al.,

*e-mail: jeduardo.unesp@yahoo.com.br

\#Este trabalho é dedicado ao Prof. Dr. Hans Viertler por ocasião do seu $70^{\circ}$ aniversário. que conseguiram separações rápidas de uma mistura não complexa de hidrocarbonetos em uma coluna tubular de vidro Pirex de $85 \mathrm{~cm}$ x $65 \mu \mathrm{m}$ (d.i.), recoberta com fase estacionária de $1 \%$ de esqualano em hexano. ${ }^{9}$

De fato, a introdução das colunas capilares foi um marco na história da cromatografia. O sucesso da utilização das colunas capilares e seu constante aprimoramento com o surgimento de novas fases estacionárias fizeram com que os problemas de resolução cromatográfica aos poucos fossem resolvidos, sendo consolidada a CG de alta resolução no final dos anos 70 e $80 .^{7}$

Entretanto, até 1980 a problemática relacionada ao aumento de velocidade em CG não estava atrelada somente a limitações relacionadas às colunas. Havia também uma série de adaptações que deveriam ser feitas nos equipamentos existentes até então, o que praticamente inviabilizava o amadurecimento da ideia. Outra grande limitação estava relacionada ao tempo total gasto em um processo analítico, no qual a separação cromatográfica não era determinante, pois representava apenas uma pequena parcela quando comparada ao tempo necessário para preparação da amostra e posterior tratamento dos dados obtidos do cromatograma. Assim, a proposta de separações rápidas em CG permanecia como um desafio experimental, visto que o aumento da velocidade da separação não causaria uma redução significativa no tempo total de análise..$^{2,10,11}$

Em decorrência da modernização dos cromatógrafos, do desenvolvimento de novas fases estacionárias e do desenvolvimento de softwares avançados para tratamentos de dados cromatográficos, naturalmente os estudos relacionados ao aumento da velocidade em CG se tornaram viáveis e receberam maior destaque no mundo acadêmico. O contínuo crescimento do número de amostras a serem analisadas por cromatografia e os casos em que os resultados das análises deveriam estar disponíveis em um curto intervalo de tempo fortaleceram esta tendência. O principal benefício seria a redução no tempo total de análise, que acarretaria em uma redução de custos no processo analítico e o consequente aumento de produtividade para realização de análises de rotina.

Em 1985, Leclercq e Cramers apresentaram os resultados de suas pesquisas direcionadas à teoria do aumento de velocidade em CG. ${ }^{12}$ 
Neste artigo os autores discutem detalhadamente a minimização do tempo de análise para solucionar problemas de separação cromatográfica com um número mínimo de pratos teóricos requeridos. Mais tarde, outros autores também apresentaram estudos teóricos relacionados à otimização da técnica. ${ }^{4,5,13-15}$ Uma abordagem mais detalhada das equações que fundamentam a técnica será apresentada adiante.

De fato, os usuários de cromatografia passaram a questionar algumas das condições experimentais convencionalmente utilizadas e chegaram à conclusão que a resolução cromatográfica poderia ser reduzida para um valor apenas suficiente (um valor limite que não causasse prejuízos para a identificação dos compostos presentes na amostra). Neste caso, haveria uma redução no tempo de análise em CG. A partir dos estudos teóricos de Cramers, dentre outros autores anteriormente citados, constatou-se que os três pilares da separação rápida são: $:^{8,12,16}$

1. Minimização da resolução cromatográfica para um valor apenas suficiente, de modo a reduzir o número de pratos teóricos requeridos para uma dada separação. Em termos práticos, duas das opções são: reduzir o comprimento da coluna e empregar altas velocidades do gás de arraste.

2. Maximização da seletividade do sistema cromatográfico: em separações rápidas, as propriedades da coluna são essenciais para melhorar a distinção entre pares críticos de componentes de uma amostra (com tempos de retenção próximos).

3. Proposição de alternativas que reduzam o tempo de análise, mantendo a resolução constante: determinados parâmetros podem ser explorados caso o tempo de análise em uma resolução mínima aceitável ainda exceda o tempo desejado. Ex: a redução do d.i. da coluna compensa a inevitável perda da resolução quando da diminuição do comprimento da coluna.

As três afirmações gerais não são independentes e o direcionamento para atingir separações rápidas resulta de uma intercalação de vários parâmetros cromatográficos, os quais serão apresentados em maiores detalhes neste trabalho.

\section{DEFINIÇÕES}

$\mathrm{O}$ aumento de velocidade em CG pode ser interpretado sob diferentes pontos de vista. Se por um lado uma separação rápida de amostras complexas pode ser obtida na faixa de alguns minutos, para uma mistura simples a separação pode ser finalizada em alguns milissegundos. ${ }^{2,5,16}$ Assim, os conceitos que fundamentam o aumento de velocidade não podem ser estabelecidos somente em relação à diferença no tempo total de análise. Além do tempo necessário para realizar a separação, outros parâmetros cromatográficos devem ser considerados para definir os conceitos relacionados ao aumento de velocidade em CG.

Dagan e Amirav ${ }^{17}$ introduziram um fator para quantificar o aumento de velocidade (Speed Enhancement Factor - SEF) e a partir deste subdividiram a CG em três categorias. Desta subdivisão provêm expressões como: cromatografia gasosa rápida (CG-R), cromatografia gasosa muito rápida (CG-MR) e cromatografia gasosa ultrarrápida (CGUR), além da convencional (CG-C). O fator de aumento de velocidade não necessariamente reflete a exata redução do tempo de análise, mas normaliza as separações em função da CG-C que, segundo os autores, é caracterizada pela utilização de coluna de $30 \mathrm{~m}$ com d.i. reduzido, de modo que o fluxo do gás de arraste na coluna seja de $1 \mathrm{~mL} \mathrm{~min}^{-1}$ (correspondente a He com velocidade linear de $34 \mathrm{~cm} \mathrm{~s}^{-1}$ ). Este fator é definido em função do produto de outros dois fatores: o fator de redução do comprimento da coluna multiplicado pelo fator que relaciona o aumento da velocidade do gás de arraste em relação à CG-C. Para os autores, a CG-UR, por exemplo, apresenta um fator de aumento de velocidade na faixa de 400 a 4000 vezes em relação à CG-C. ${ }^{17}$
Van Deursen et al. ${ }^{18}$ sugeriram uma definição baseada na largura do pico à meia altura e no tempo total de análise. Neste caso, considerando a largura do pico, seria considerado também o grau de separação por tempo de cada componente da amostra. Nesta classificação as separações, realizadas em CG-UR, estariam limitadas à faixa dos subssegundos e largura dos picos de aproximadamente $10 \mathrm{~ms}$.

Blumberg e Klee adotaram uma forma objetiva para avaliar o aumento de velocidade e classificaram os diferentes tipos de CG com base na largura dos picos cromatográficos. ${ }^{19}$ Mais tarde, Magni et al. apresentaram uma definição mais detalhada, divergindo das anteriores. ${ }^{19}$ Segundo esta definição, outros fatores como as dimensões da coluna e a taxa de aquecimento também devem ser considerados para diferenciar as modalidades de CG. De acordo com a Tabela 1, separações ultrarrápidas somente seriam obtidas em colunas com dimensões bastante reduzidas e com altas taxas de aquecimento, resultando em picos com largura entre 0,05 e $0,2 \mathrm{~s}{ }^{19}$

Tabela 1. Principais parâmetros que caracterizam as diferentes modalidades de cromatografia gasosa segundo Magni et al.. Adaptada da ref. 19

\begin{tabular}{lcccc}
\hline $\begin{array}{l}\text { Classificação segundo } \\
\text { Magni } \text { et al. }{ }^{19}\end{array}$ & CG-UR & CG-R & CG-CC* & CG-C \\
\hline $\begin{array}{l}\text { Comprimento coluna } \\
(\mathrm{m})\end{array}$ & 2 a 10 & 5 a 15 & 5 & 25 a 30 \\
$\begin{array}{l}\text { Diâmetro interno } \\
(\mathrm{mm})\end{array}$ & 0,05 a 0,10 & 0,10 a 0,25 & 0,25 & 0,25 a 0,32 \\
$\begin{array}{l}\text { Tempo de análise } \\
(\text { min) }\end{array}$ & $\leq 1$ & $\leq 10$ & 3 a 15 & 10 a 60 \\
$\begin{array}{l}\text { Taxa de aquecimento } \\
\left({ }^{\circ} \text { C/min) }\right.\end{array}$ & $\geq 60$ & 15 a 60 & 5 a 40 & 1 a 10 \\
Largura pico (s) & 0,05 a 0,2 & 0,5 a 2 & 1 a 5 & 1 a 10 \\
\hline
\end{tabular}

*Cromatografia gasosa com coluna curta.

Dentre todas as modalidades de CG, a CG-UR proporciona o maior fator de aumento de velocidade quando comparada à CG convencional, visto que o tempo total de análise pode ser reduzido para o limite de poucos minutos ou menos.

Embora os termos CG-UR e CG-R, dentre outros, sejam usualmente encontrados na literatura, na verdade não há uma posição acadêmica consolidada a respeito dessas classificações. Tais divergências permitem uma visão bastante subjetiva por parte dos usuários de cromatografia no momento de classificar o tipo de modalidade utilizada em seus trabalhos, principalmente em relação à CG-UR.

\section{PRINCIPAIS PARÂMETROS ENVOLVIDOS NA REDUÇÃO DO TEMPO DE ANÁLISE}

Em cromatografia gasosa a eficiência do processo de separação é influenciada por uma série de fatores, dentre os quais se pode citar: tempo de retenção, comprimento da coluna, temperatura da coluna, pressão do gás de arraste, complexidade da amostra, técnica de injeção, entre outros. ${ }^{6,20}$ Atenção especial deve ser dada a tais parâmetros quando se pretende aumentar a velocidade de uma separação cromatográfica, de modo que a resolução cromatográfica possa ser reduzida a um valor mínimo, encurtando o tempo de análise sem, no entanto, comprometer a identificação dos pares críticos de componentes da amostra.

Leclercq e Cramers partiram de uma série de deduções matemáticas para relacionar o tempo de análise em separações cromatográficas com os parâmetros anteriormente citados. ${ }^{12}$ Os autores conseguiram chegar a uma equação final do tempo de análise (Equação 1) em CG tomando como ponto de partida a equação de pratos teóricos, de 
Golay-Giddings, e a equação de relação de fluxo, de Hagen-Poiseuille, ambas para colunas capilares: ${ }^{12,16}$

$$
t_{R}=(1+k) N_{\text {req }}^{3 / 2} \frac{9}{8} \sqrt{3} F(k)\left[\frac{\eta}{p_{o} D_{m . o}}\right]^{1 / 2} d_{c}
$$

onde $t_{R}$ é o tempo de retenção, $k$ é o fator de retenção, $F$ é dada em função do fator de retenção, $n$ é a viscosidade dinâmica, $p_{o}$ é a pressão na saída do sistema, $D_{m, o}$ é o coeficiente de difusão do soluto na fase móvel em relação à pressão na saída, $d_{c}$ é o d.i. da coluna capilar. $\mathrm{O}$ número de pratos teóricos requeridos para a separação, $N_{\text {req }}$, é dado em função do fator de separação $(\alpha)$ de dois picos, do fator de retenção e, principalmente, em função da resolução $\left(R_{s}\right)$ para um par crítico de componentes da amostra.

De acordo com a Equação 1, reduções significativas no tempo de análise podem ser obtidas, principalmente, com a utilização de colunas de dimensões reduzidas e com o aumento da pressão ou aplicação de vácuo na saída do sistema. ${ }^{12,16}$ Ainda com base nesta equação, há uma relação direta entre $t_{R}$ e o termo referente à fase gasosa: $\mathrm{t}_{\mathrm{R}} \propto\left[n / p_{o} D_{m, o}\right]$. Este termo tem um valor mínimo para o hidrogênio, que apresenta menor viscosidade e maior coeficiente de difusão quando comparado (na mesma temperatura) com outros gases usualmente utilizados em CG, tais como hélio e nitrogênio. Além disso, um coeficiente de difusão relativamente alto confere ao hidrogênio um desempenho melhor do que os outros gases quando são utilizadas velocidades lineares maiores. ${ }^{4,12,21}$

Aproximações matemáticas diferentes evidenciam também a influência de outros parâmetros, conforme a Equação $2:^{4}$

$$
t_{R}=36\left[\left(\frac{(1+k)^{3}}{k^{2}}\right)\left(\frac{\alpha}{\alpha-1}\right)^{2}\right]\left[\frac{H}{u_{o} f_{2}}\right]
$$

onde 36 é o valor constante para separação do par crítico com resolução de 1,5 na linha de base, $H$ é a altura equivalente a um prato teórico, $u_{o}$ é a velocidade linear do gás de arraste na saída da coluna e $f_{2}$ é o fator de correção da compressibilidade do gás de arraste. ${ }^{4}$

Os autores ainda estenderam seus estudos a respeito da redução do tempo de análise, propondo novas equações matemáticas. Segundo esses estudos, todas as deduções usadas até chegar às equações finais mostram uma complexa dependência do tempo de análise com a pressão, de modo que as condições ótimas para estas equações são aproximadas e, assim, puramente matemáticas. Relações explícitas só poderiam ser obtidas sob condições extremas, quando as razões de pressão de entrada e saída do sistema forem muito baixas ou muito altas ou ainda quando a influência da fase estacionária for negligenciável. ${ }^{4,5}$

Na modalidade CG-UR também deve ser considerado que a determinação de ótimas taxas de aquecimento têm influência direta na qualidade da separação e velocidade da análise. Assim, não somente altas taxas de aumento de temperatura, como também curtos períodos de resfriamento e retomada da temperatura da coluna devem ser levados em consideração para a realização de análises mais rápidas. ${ }^{22-25}$

\section{CARACTERÍSTICAS DO SISTEMA CG-UR}

O aumento de velocidade não necessariamente dependerá de substituição ou mesmo adaptação dos equipamentos convencionais de CG, mas nem sempre os equipamentos convencionais são compatíveis com a velocidade requerida. Para propor uma redução de duas a quatro vezes no tempo total necessário para realizar uma análise cromatográfica, a utilização de colunas de dimensões reduzidas acompanhada por simples alterações feitas em alguns parâmetros cromatográficos, tais como aumento na taxa de aquecimento e otimização da velocidade do gás de arraste, podem ser suficientes. Por outro lado, separações ultrarrápidas, que chegam a promover uma redução de dez ou mais vezes no tempo total de análise podem requerer uma adaptação significante dos cromatógrafos convencionais. Neste caso, amostradores automáticos de alta velocidade, sistemas para rápido aquecimento da coluna e detectores com altas taxas de aquisição de dados são itens obrigatórios. ${ }^{11,24,26}$

\section{Amostradores e injetores}

Aparentemente, os amostradores e injetores são os mesmos utilizados em CG-C. São preferíveis os amostradores automáticos de alta velocidade para diminuir o máximo possível o tempo necessário para completar um ciclo analítico - tempo total entre uma injeção e outra, incluindo resfriamento da coluna e tempo de amostragem. Dos injetores convencionais, em princípio todos poderiam ser aplicados em CG-UR: Split/Splitless, Programmable Temperature Vaporizer - PTV, ${ }^{17,19,25,27-30}$ além do sistema de injeção direto na coluna - Cold On-Column. ${ }^{31,32} \mathrm{O}$ fator limitante do sistema Cold On-Column está relacionado ao d.i. da coluna analítica que, em muitos casos, torna obrigatória a conexão desta com uma pré-coluna de maior d.i.. ${ }^{8} \mathrm{O}$ sistema com amostrador em headspace também já foi aplicado na modalidade ultrarrápida. ${ }^{18}$

A injeção de volumes em escala nanométrica pode ser uma ferramenta muito útil em sistemas ultrarrápidos, nos quais são usadas colunas com menor capacidade de processamento da amostra (d.i. reduzido). Neste caso, seriam necessárias pequenas adaptações nos amostradores automáticos utilizados em CG-C como, por exemplo, a utilização de seringas com êmbolo na agulha (plunger-in-needle syringe). ${ }^{33,34}$

\section{Coluna cromatográfica e sistema de aquecimento da coluna}

As colunas capilares utilizadas em CG-UR possuem dimensões reduzidas, em geral com comprimento variando de 2 a $10 \mathrm{~m}$, d.i. de 0,05 a 0,1 mm e espessura do filme da fase estacionária a partir de 0,05 $\mu \mathrm{m}$, conforme mostra a Tabela 1 . Colunas de dimensões reduzidas são fundamentais para a diminuição no tempo de análise e obtenção de cromatogramas bem resolvidos, constituindo o primeiro passo em direção a separações ultrarrápidas. ${ }^{4,8,15,16,18,19,28,35}$

A variação do d.i. em uma coluna cromatográfica promove um balanço entre dois fatores: a eficiência da coluna (determinada pelo número de pratos teóricos - N) e a capacidade da coluna (relacionada à quantidade de amostra que pode acomodar a coluna sem causar distorção ou perda de resolução dos picos). A otimização de um destes fatores requer o sacrifício do outro, de modo que o balanço entre os dois vai depender das necessidades analíticas. Em se tratando de cromatografia gasosa ultrarrápida, a utilização de colunas com d.i. reduzido (narrow bore columns) proporciona aumento no número de pratos teóricos, gerando picos mais estreitos, com larguras inferiores a 0,2 s, e surge como alternativa para compensar a perda de resolução em decorrência da diminuição do comprimento da coluna. ${ }^{4,11,15,36,37}$

Separações ultrarrápidas são facilitadas também com a utilização de colunas com fino depósito de fase estacionária (Tabela 1), onde a resistência oferecida no processo de transferência de massa nesta fase é praticamente insignificante quando comparada àquela da fase gasosa. ${ }^{4,15}$

Com relação às altas taxas de programação de temperatura da coluna usadas em CG-UR, uma das opções é a utilização de sistemas dedicados ao aquecimento, no qual a coluna do tipo narrow bore é colocada em um módulo ultrarrápido (ultra fast module - UFM), conhecido como "gaiola". Em uma das concepções deste sistema a coluna é montada com o elemento aquecedor (resistência elétrica) e com o sensor de temperatura, todos envolvidos por um revestimento 
de teflon (ou fibra cerâmica) constituindo um conjunto de pouca massa térmica - Figura 1 . $^{19,28,29,38,39}$

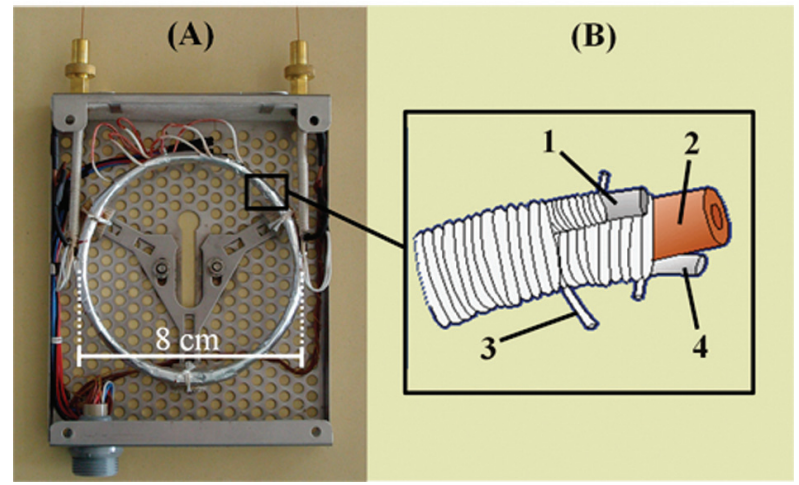

Figura 1. (A) Sistema dedicado de aquecimento resistivo da coluna (Thermo Fisher Scientific Inc. $\left.{ }^{\circledR}\right)$. (B) Detalhe do revestimento da coluna capilar: 1- elemento aquecedor; 2- coluna capilar; 3-fibra cerâmica; 4- sensor de temperatura

Desta forma, nenhum aquecimento do forno é exigido e são utilizadas as interfaces convencionais de injetor e detector do cromatógrafo convencional (Figura 2). O sistema modular para o aquecimento resistivo direto da coluna permite alcançar maiores velocidade de aquecimento quando comparado ao forno cromatográfico convencional de circulação a ar. Em se tratando de CG-UR este módulo é imprescindível para a obtenção de separações cromatográficas no limite de alguns segundos. ${ }^{19,28,40}$

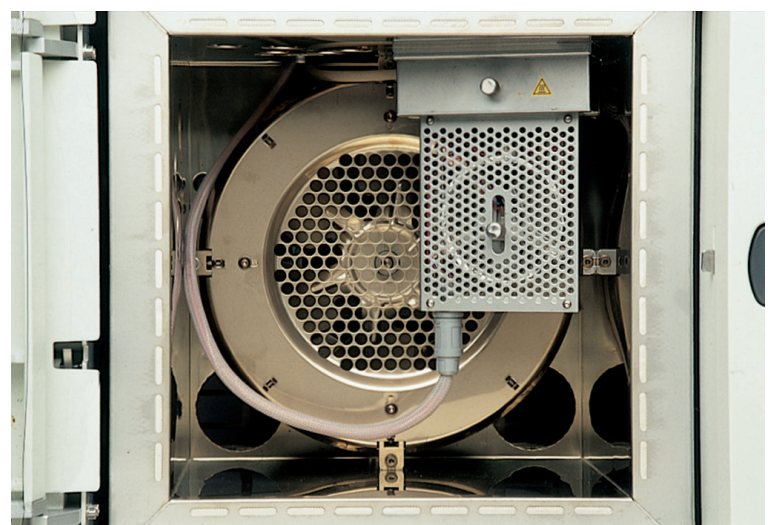

Figura 2. Módulo de aquecimento ultrarrápido instalado no forno de um cromatógrafo convencional (Thermo Fisher Scientific Inc. ${ }^{\circledR}$ )

A ideia do aquecimento resistivo direto da coluna surgiu na década de 60 , mas teve sua eficácia experimental comprovada somente a partir dos anos 90, quando diferentes propostas deste sistema foram publicadas na literatura. ${ }^{40-50}$

A partir do trabalho de Ehrmann et al. ${ }^{40}$ e das patentes de Mustacich et al.$^{47-49} \mathrm{e}$ Magni et al. ${ }^{50}$ foram concebidos os diferentes módulos de aquecimento ultrarrápidos comercializados atualmente, projetados para comportar colunas de vários comprimentos e que podem ser instalados tanto em cromatógrafos convencionais quanto em sistemas portáteis de análise cromatográfica. Esses módulos de aquecimento resistivo da coluna permitem atingir taxas de aquecimento de até $20{ }^{\circ} \mathrm{C} \mathrm{s}^{-1}$, sendo que o resfriamento da coluna é rápido, indo de 350 a $50{ }^{\circ} \mathrm{C}$ em cerca de $1 \mathrm{~min}$. Por não necessitar do aquecimento do forno do cromatógrafo, o consumo de energia é menor (200 W) quando comparado ao modo convencional. ${ }^{44-46,51}$
Em CG, a temperatura afeta de forma significativa os aspectos cinéticos e termodinâmicos relacionados ao equilíbrio existente entre a fase móvel-analito-fase estacionária, alterando, dentre outros parâmetros citados nas Equações 1 e 2, a seletividade da fase estacionária e o espalhamento das zonas cromatográficas, provocando, consequentemente, variações no desvio padrão dos tempos de retenção dos componentes da amostra. Deste modo, quando altas taxas de aquecimento são necessárias para promover separações ultrarrápidas, o programa de temperatura deve ser controlado com grande precisão. ${ }^{22,42,52}$

\section{Detectores}

A alta velocidade com que os componentes separados na coluna chegam ao detector é um fator importante a ser considerado em CGUR, de modo que a eficiência da separação da coluna não seja comprometida pelo tempo de resposta do detector. O processamento de bandas cromatográficas com largura de $50 \mathrm{~ms}$ na linha de base requer detectores com alta frequência de amostragem, acima de $200 \mathrm{~Hz} .{ }^{16,53}$

Quanto maior a frequência de aquisição, maior será a capacidade do detector para responder às mudanças de sinal provenientes das substâncias que eluem da coluna. Dentre os detectores usualmente empregados em CG, os detectores de ionização em chama (DIC) e espectrômetro de massas (EM) são, de longe, os mais utilizados na modalidade ultrarrápida, sendo que o primeiro é facilmente encontrado nos modernos equipamentos de CG com frequência de aquisição de dados de até $300 \mathrm{~Hz}$. ${ }^{19,28}$

O detector de massas torna-se especialmente vantajoso quando são utilizados sistemas sem varredura total da amostra (Non-Scanning), tal como os espectrômetros de massas com analisadores por tempo de vôo (ATV), ${ }^{35}$ ainda que alguns analisadores quadrupolo venham sendo utilizados. ${ }^{51}$ Um sistema EM-ATV pode processar aproximadamente 500 espectros completos por segundo e proporciona exatidão na detecção de picos com largura na ordem dos milissegundos, sem distorção dos dados espectrais. ${ }^{16,35}$ Em contrapartida, a alta taxa de aquisição de dados é acompanhada pela perda da sensibilidade em ATV, que pode ser contornada com a geração de feixes moleculares supersônicos (Sonic Molecular Beam - SMB). Os sistemas SMB apresentam bom desempenho em CG-UR, pois requerem altas taxas de fluxo em sua interface para gerar o feixe supersônico que, por sua vez, provoca uma queda de energia térmica no sistema, intensificando o sinal do íon molecular. 15,17,27,35,57-59

A alta velocidade com que os cromatogramas são obtidos em CG-UR muitas vezes exige que compostos coeluídos sejam adequadamente resolvidos no detector EM com o auxílio de programas de deconvulação (deconvolution software). São programas que usam algoritmos matemáticos para inverter os efeitos de entrelaçamento de dados e são capazes de identificar componentes individuais em uma amostra minimamente separada na fase cromatográfica. Muitos dos trabalhos que envolvem CG-UR encontrados na literatura lançam mão desta estratégia para auxiliar na identificação dos componentes da amostra. ${ }^{27,35,51}$

A utilização de outros detectores, como o nitrogênio-fósforo (DNF), fotométrico de chama (DFC), fotoionização (DFI), captura de elétrons (DCE) e condutividade térmica (DCT) vai depender da frequência de aquisição dos mesmos e da configuração física interna de seus dispositivos. ${ }^{54-56}$ Apesar de serem dotados de eletrônica rápida, alguns destes detectores, por exemplo, o DCT e DFI, ainda apresentam células com grande volume interno e acabam não sendo adequados para a realização de análises ultrarrápidas.

\section{CAMPOS DE APLICAÇÃO}

A CG-UR pode ser empregada para realização de análises qualitativas e quantitativas em todos os campos de aplicação da cro- 
matografia capilar, sendo especialmente interessante nos casos em que a amostra contém componentes com pontos de ebulição muito diferentes. Vários trabalhos têm sido publicados com a utilização da técnica, sendo que alguns destes serão apresentados com maiores detalhes a seguir. ${ }^{2,17-19,25,27-32,38,39,43}$

Em 1982, Jonker et al. conseguiram obter a separação de nalcanos (C1-C4) naquela que viria a ser a separação cromatográfica mais rápida da época utilizando colunas empacotadas, finalizada em menos de $0,15 \mathrm{~s}$. As condições cromatográficas utilizadas foram: coluna empacotada: 32 x 1,19 mm d.i.; $10 \mu \mathrm{m}$ de tamanho de partícula, Lichrosorb Si-60; pressão de entrada 64 bar; gás de arraste hélio, 1,5 $\mu \mathrm{L}$ injetado no modo splitless. ${ }^{2}$

Em se tratando de amostras complexas, Dagan e Amirav ${ }^{17}$ discutiram a capacidade de um sistema CG-EM em realizar análises de classes diferentes de substâncias químicas, em diferentes velocidades (CG-R; CG-MR; CG-UR). O principal objetivo era diminuir o tempo de residência das moléculas termolábeis na coluna e assim minimizar seu grau de dissociação que, neste caso, poderia ocasionar o alargamento de algumas bandas ou, ainda, provocar o aparecimento de novas bandas cromatográficas com tempo de retenção menores. Sete esteroides não derivados foram analisados e, dentre estes, a cortisona, a corticosterona e o $\beta$-estradiol puderam ser quantificados somente por cromatografia gasosa ultrarrápida, já que nas modalidades rápida e muito rápida essas moléculas sofriam dissociação. Para a modalidade ultrarrápida, as condições cromatográficas foram: gás de arraste hélio a $500 \mathrm{~cm} / \mathrm{s}$, temperatura do injetor a $280{ }^{\circ} \mathrm{C}$ para uma coluna megabore de $50 \mathrm{~cm}$ x $0,53 \mathrm{~mm}$ d.i. a $235^{\circ} \mathrm{C}$. A separação foi finalizada em apenas $8 \mathrm{~s}$, com esperada perda de resolução. Para contornar essa perda de resolução, a espectrometria de massas com ionização eletrônica em SMB foi utilizada, favorecendo a sintonização da massa de cada fragmento, minimizando os problemas de superposição dos picos e permitindo a identificação e quantificação dos componentes. ${ }^{17}$

No mesmo trabalho, os autores conseguiram realizar a separação de alguns fármacos e de pesticidas da classe dos carbamatos também por CG-UR, ressaltando a utilização da técnica para outros tipos de substâncias instáveis ou termolábeis. Como no caso anterior, apesar da perda de resolução quando comparada às modalidades rápida e muito rápida, os autores justificaram a opção pela modalidade ultrarrápida devido aos problemas de dissociação que impediam a quantificação. ${ }^{17}$

Davis et al. reportaram a separação de 7 solventes de laboratório por CG-UR, com a utilização de coluna de sílica fundida com $1 \mathrm{~m}$ x $0,1 \mathrm{~mm}$ d.i. $(0,1 \mu \mathrm{m}$ de espessura de filme). Para identificação dos analitos foi utilizada a técnica de espectrometria de massas com ionização de impactos de elétrons em SMB e ATV para contornar os problemas de resolução cromatográfica. ${ }^{27}$

Van Deursen et al. utilizaram o sistema de aquecimento resistivo direto da coluna para obter separações ultrarrápidas de 10 diferentes hidrocarbonetos por cromatografia gasosa. Neste trabalho, também foram relatados problemas de sobreposição de picos cromatográficos, sendo necessária a utilização de um programa de deconvulação para realizar a identificação de tais picos por espectrometria de massas. ${ }^{18}$

Bicchi et al. conseguiram obter separações ultrarrápidas de óleos essenciais de diferentes complexidades. Os pares mais críticos dos componentes de cada amostra foram separados após um estudo detalhado para se escolher a fase estacionária mais seletiva, a fim de compensar a perda de resolução devido ao uso de colunas curtas e altas taxas de aquecimento. Com este estudo e com a utilização de detector DIC com alta frequência de aquisição de dados $(300 \mathrm{~Hz}-$ aquisições a cada $6 \mathrm{~ms}$ ) os cromatogramas obtidos apresentaram boa resolução para identificação das espécies. A Figura 3 mostra que praticamente não há diferença no perfil cromatográfico quando são comparados os cromatogramas do óleo essencial de sálvia obtidos por CG-C e CG-UR. ${ }^{19}$
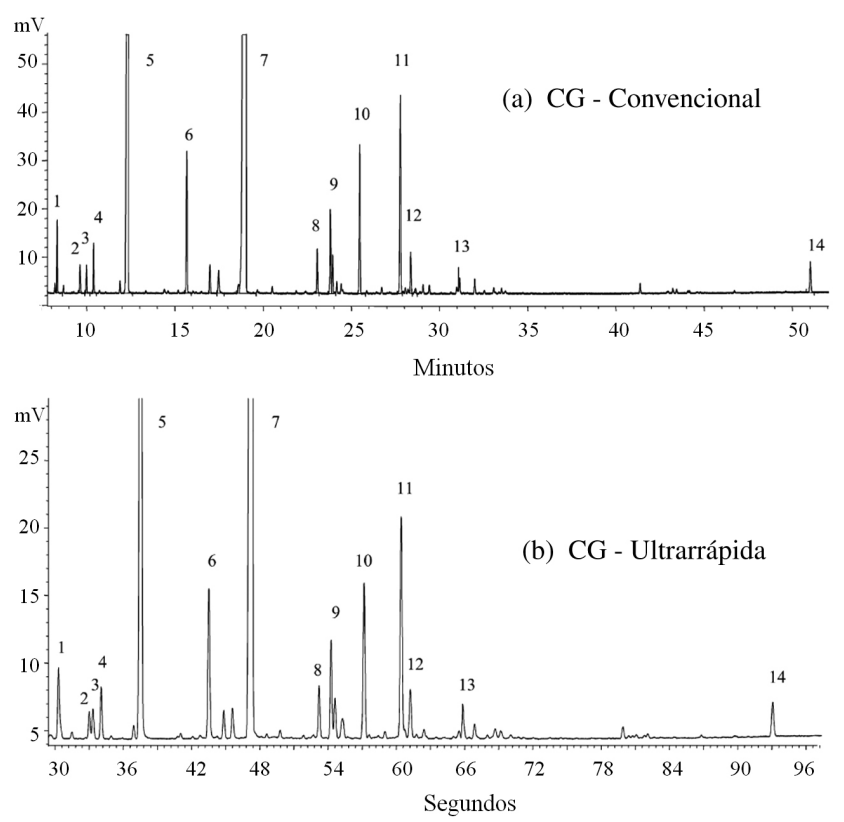

Figura 3. Perfis cromatográficos obtidos para o óleo essencial de sálvia por CG-C (a) e CG-UR (b). Condições cromatográficas: (a) coluna SE 54: $25 \mathrm{~m}$; 0,25 d.i.; 0,3 $\mu \mathrm{m}$; razão de split: $30 ; \mathrm{H}_{2}$ a 47,8 $\mathrm{cm} \mathrm{s}^{-1}$; programação de temperatura: $50{ }^{\circ} \mathrm{C}(1 \mathrm{~min}) ; 3{ }^{\circ} \mathrm{C} \mathrm{min}^{-1}$ até $250{ }^{\circ} \mathrm{C}$. (b) coluna SE 54: 5 m; 0,1 d.i.; 0,1 $\mu \mathrm{m}$; razão de split: 300; $\mathrm{H}_{2}$ a 69,3 $\mathrm{cm} \mathrm{s}^{-1}$; programação de temperatura: $50^{\circ} \mathrm{C}(0,1 \mathrm{~min}) ; 150^{\circ} \mathrm{C} \mathrm{min}^{-1}$ até $250^{\circ} \mathrm{C}$. Adaptada de ref. 19

Praticamente na mesma época, Facchetti e Cadopi também relataram a possibilidade da separação de diferentes classes de óleos essenciais com a utilização de diferentes tipos de fases estacionárias. Para todas as classes de óleos os autores realizaram comparação dos dados obtidos por CG-UR e CG-C e constataram reduções de aproximadamente $30-40$ vezes no tempo de análise. Foi avaliada ainda a repetibilidade do sistema ultrarrápido para o óleo de lavanda em coluna OV5 5 m x 0,1 mm d.i. x 0,1 $\mu$ m, enfatizando a identificação inequívoca dos componentes do óleo essencial. ${ }^{29}$ Os autores também aplicaram a CG-UR na determinação de hidrocarbonetos de petróleo em água potável e solo. Além da redução de aproximadamente 5 vezes no tempo de análise, o método ultrarrápido foi mais sensível quando comparado ao método convencional de análise. ${ }^{38}$

Em outro trabalho, as normas internacionais ASTM D $6584^{60} \mathrm{e}$ EN $14105^{61}$ foram adaptadas para a determinação ultrarrápida dos teores de glicerina livre e total em biodiesel puro (B100). Para isso foi realizada uma série de alterações nos parâmetros cromatográficos recomendados em cada uma das normas, de modo a reduzir em mais de 6 vezes o tempo de análise, que era de aproximadamente 30 min. Dentre as alterações necessárias, a principal foi a inclusão do módulo de aquecimento resistivo (Figuras 1 e 2) que possibilitou alcançar uma razão de aquecimento de $80^{\circ} \mathrm{C} \mathrm{min}{ }^{-1}$. A Figura 4 mostra o cromatograma obtido, bem como as condições cromatográficas utilizadas. Os autores, entretanto, não relataram a velocidade do gás de arraste utilizada. ${ }^{32}$

Krokosová et al. propuseram outro sistema de aquecimento capaz de atingir rápidas programações de temperatura. Neste sistema, a coluna é recoberta por tubos coaxiais de aço inoxidável, preenchidos com óleo parafínico de viscosidade e estabilidade térmica apropriados. Em uma das aplicações do sistema desenvolvido pelos autores, várias substâncias pertencentes à classe dos fenóis foram separadas e reveladas por DIC em menos de 1 min, evidenciando que a resolução obtida possibilitou a identificação de todos os componentes presentes em uma mistura de 11 substâncias fenólicas. ${ }^{25}$ 


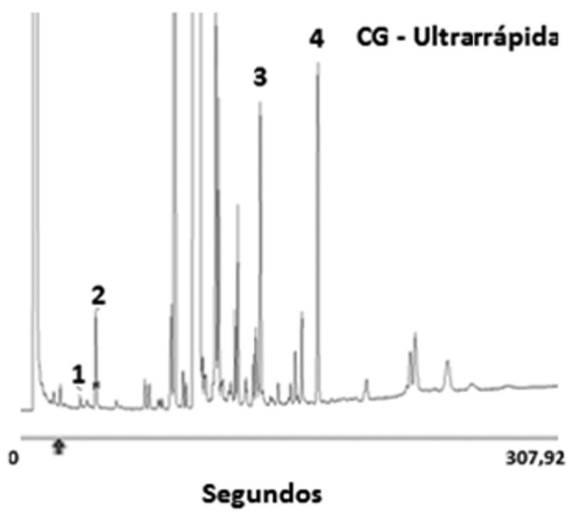

Figura 4. Cromatograma obtido na análise dos teores de glicerina livre e total em amostras de biodiesel. 1- Glicerol, 2-Butanotriol (padrão interno), 3-Mono-oleína, 4-Tricaprina (padrão interno). Condições cromatográficas: coluna SE-54 com $3 \mathrm{~m} \times 0,32 \mathrm{~mm}$ d.i. $\times 0,05$ m e sistema de injeção no modo PTV/On-Column. A programação de temperatura adotada foi: $85{ }^{\circ} \mathrm{C}$ por 0,3 $\min ; 80^{\circ} \mathrm{C} \mathrm{min}^{-1}$ até $270{ }^{\circ} \mathrm{C}^{32}$

Povolo et al. aplicaram a CG-UR no estudo da pureza de gorduras (triacilglicerois) presentes em amostras de leite de vaca. A potencialidade da técnica para este tipo de aplicação foi ressaltada pelos autores, que obtiveram um desvio padrão relativo (DPR) abaixo dos $2 \%$ para praticamente todos os triacilglicerois presentes na amostra. A Figura 5 traz uma comparação entre os cromatogramas obtidos por CG-C e CG-UR e as condições em que as análises foram realizadas. ${ }^{31}$
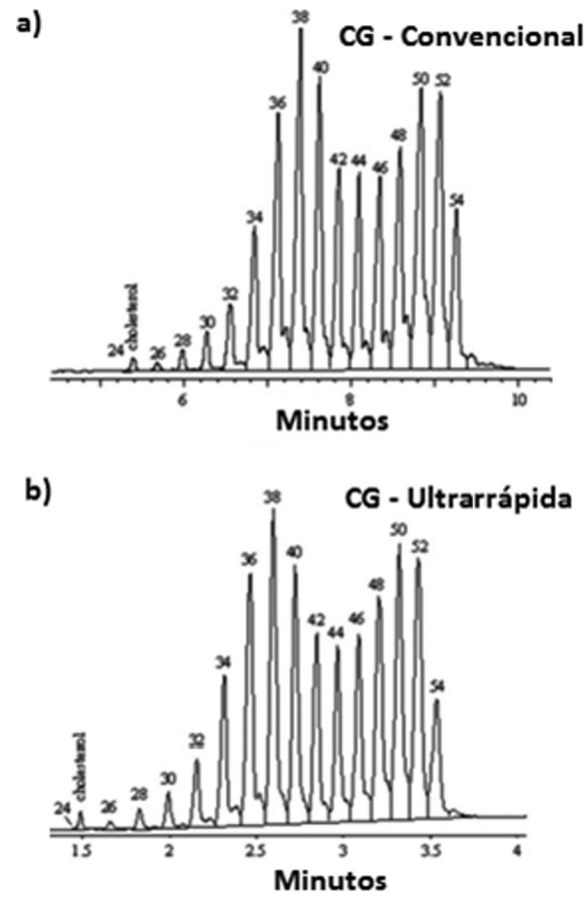

Figura 5. Comparação entre as modalidades CG-C e CG-UR. (a) coluna

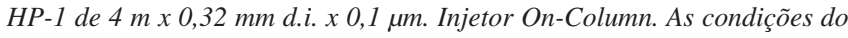
forno foram: $60^{\circ} \mathrm{C}(2 \mathrm{~min}) ; 35^{\circ} \mathrm{C} \mathrm{min}^{-1}$ até $340{ }^{\circ} \mathrm{C}(2 \mathrm{~min})$. Gás de arraste: hidrogênio a $5 \mathrm{~mL} \mathrm{~min}{ }^{-1}$ (fluxo constante). DIC a $350^{\circ} \mathrm{C}$. (b) Coluna HP-1 de 2,5 m $x$ 0,32 mm d.i. $\times 0,05 \mu \mathrm{m}$. Injetor PTV/Split (1:30). Programação de temperatura: $150{ }^{\circ} \mathrm{C}(0,5 \mathrm{~min}) ; 60^{\circ} \mathrm{C}$ min $^{-1}$ até $340{ }^{\circ} \mathrm{C}(0,5 \mathrm{~min})$. Gás de arraste: hidrogênio a $3 \mathrm{~mL} \mathrm{~min}^{-1}$ (fluxo constante). DIC de alta frequência a $350{ }^{\circ} \mathrm{C}$. Os números de 24 a 54 correspondem ao tamanho da cadeia carbônica dos triacilglicerois encontrados na gordura do leite. Adaptada de ref. 31
Ficarra et al. estudaram a performance de 4 catalisadores $\left(\mathrm{NaOCH}_{3}, \mathrm{BF}_{3}, \mathrm{H}_{2} \mathrm{SO}_{4}, \mathrm{KOH}\right)$ para realizar a transesterificação de gordura animal proveniente do tecido adiposo subcutâneo de suínos. O rendimento da reação de transesterificação foi obtido pela determinação do perfil composicional de ésteres metílicos de ácidos graxos por CG-UR. A separação completa de todos os ésteres presentes na amostra foi realizada em pouco mais de 2 min, utilizando-se o módulo de aquecimento resistivo (Figuras 1 e 2). As condições cromatográficas utilizadas foram: coluna Carbowax de $5 \mathrm{~m} \mathrm{x} \mathrm{0,1} \mathrm{mm} \mathrm{d.i.} \mathrm{x} \mathrm{0,2}$ $\mu \mathrm{m}$ (envolta pelo módulo ultrarrápido). Injetor PTV/Split (1:150) a $240{ }^{\circ} \mathrm{C}$. Programação de temperatura: $150{ }^{\circ} \mathrm{C}(10 \mathrm{~s}) ; 102{ }^{\circ} \mathrm{C} \mathrm{min}{ }^{-1}$ até $240{ }^{\circ} \mathrm{C}$ (2,3 min). Foi utilizado hélio como gás de arraste a $0,5 \mathrm{~mL}$ $\min ^{-1}$ (fluxo constante). DIC de alta frequência a $240{ }^{\circ} \mathrm{C} .{ }^{30}$

\section{CONCLUSÃO}

Em princípio, a cromatografia a gás ultrarrápida pode ser empregada em todos os campos de aplicação da cromatografia gasosa convencional, mas com algumas ressalvas. $\mathrm{O}$ acentuado aumento de velocidade alcançado pelo aumento da pressão, pela utilização de colunas com dimensões reduzidas e por altas taxas de aquecimento geralmente é acompanhado pela concomitante perda de resolução. No caso de amostras pouco complexas, a resolução pode ser facilmente diminuída a um valor mínimo exigido, sem que haja problemas na identificação de pares críticos de componentes na amostra, ao passo que na separação de amostras mais complexas por CG-UR a perda de resolução pode dificultar a identificação desses pares críticos.

Dentre os trabalhos reportados na literatura, que fazem menção à aplicação desta modalidade de cromatografia, quase todos lançam mão de alguma estratégia para contornar os problemas de resolução cromatográfica de amostras complexas. Essas estratégias vão desde a utilização da EM-ATV e sistemas SMB para auxiliar a interpretação de cromatogramas com resolução insatisfatória, até mesmo ao emprego de programas específicos de deconvulação para realizar o tratamento de dados. Uma solução mais condizente com os preceitos cromatográficos seria a realização de um estudo prévio detalhado, para escolher a fase estacionária de maior seletividade para a amostra a ser analisada por CG-UR.

Enfim, os principais conceitos relacionados à CG-UR, assim como alguns aspectos técnicos referentes à instrumentação e acessórios utilizados, ainda podem ser exaustivamente explorados a fim de tornar viável a aplicação desta modalidade de CG em análises de rotina.

\section{AGRADECIMENTOS}

Aos representantes da Thermo Fisher Scientific Inc. por cederem imagens referentes ao módulo UFM-GC; aos apoios financeiros da FUNDUNESP (Fundação para o Desenvolvimento da Unesp), CAPES (Coordenação de Aperfeiçoamento de Pessoal de Nível Superior) e do CNPq (Conselho Nacional de Desenvolvimento Científico e Tecnológico) às pesquisas, na forma de auxílios e bolsas.

\section{REFERÊNCIAS}

1. Dandeneau, R.; Hawkes, S.; Chromatographia 1980, 13, 686.

2. Jonker, R. J.; Poppe, H.; Huber, J. F. K.; Anal. Chem. 1982, 14, 2447.

3. Lewis, D. A.; Vouros, P.; Karger, B. L.; Chromatographia 1982, 15, 117.

4. Cramers, C. A.; Leclercq, P. A.; J. Chromatogr., A 1999, 842, 3.

5. Cramers, C. A.; Janssen, H.; van Deursen, M. M.; Leclercq, P. A.; J. Chromatogr., A 1999, 856, 315.

6. Collins, C. H.; Braga, G. L.; Bonato, P. S.; Fundamentos de Cromatografia, UNICAMP: Campinas, 2006; Lanças, F. M.; Cromatografia Gasosa, Acta: São Carlos, 1993. 
7. Pereira, A. S.; Aquino Neto, F. R.; Quim. Nova 2000, 23, 370.

8. Matisová, E.; Domotorová, M.; J. Chromatogr., A 2003, 1000, 199.

9. Gaspar, G.; Vidal-Madjar, C.; Guiochon, G.; Chromatographia 1982, $15,125$.

10. Ettre, L. S.; $L C G C$ 2001, 19, 120.

11. Hinshaw, J. V.; LCGC 2001, 19, 170.

12. Leclercq, P. A.; Cramers, C. A.; J. High Resolut. Chromatogr. Chromatogr. Commun. 1985, 8, 764.

13. Blumberg, L. M.; J. High Resol. Chromatogr. 1997, 20, 597.

14. Blumberg, L. M.; J. High Resol. Chromatogr. 1997, 20, 679.

15. Leclercq, P. A.; Cramers, C. A.; Mass Spectrom. Rev. 1998, 17, 37.

16. Korytár, P.; Janssen, H. G.; Matisová, E.; Brinkman, U. A.; Trends Anal. Chem. 2002, 21, 558.

17. Dagan, S.; Amirav, A.; J. Am. Soc. Mass Spectrom. 1996, 7, 737.

18. van Deursen, M.; Beens, J.; Janssen, H. G.; Leclercq, P. A.; Cramers, C. A.; J. Chromatogr., A 2000, 878, 205.

19. Bicchi, C.; Brunelli, C.; Cordero, C.; Rubiolo, P.; Galli, M.; Sironi, A.; J. Chromatogr., A 2004, 1024, 195.

20. Lanças, F. M.; McNair, H. M.; Quim. Nova 1983, 6, 6.

21. http://chromatographyonline.findanalytichem.com/lcgc/article/ articleDetail.jsp?id=564647, acessada em Outubro 2010.

22. Blumberg, L. M.; Klee, M. S.; J. Microcolumn Sep. 2000, 12, 508.

23. Blumberg, L. M.; Klee, M. S.; Anal. Chem. 2000, 72, 4080.

24. Dorman, F. L.; Overton, E. B.; Whiting, J. J.; Cochran, J. W.; GardeaTorresdey, J.; Anal. Chem. 2008, 80, 4487.

25. Krkosová, Z.; Kubinec, R.; Jurdakova, H.; Blasko, J.; Ostrovski, I.; Sojak, L.; Sevcik, J.; Chem. Pap. 2008, 62, 135.

26. Eiceman, G. A.; Gardea-Torresdey, J.; Overton, E.; Carney, K.; Dorman, F.; Anal. Chem. 2004, 76, 3387.

27. Davis, S. S.; Makarov, A. A.; Hughes, J. D.; Rapid Commun. Mass Spectrom. 1999, 13, 237.

28. Bicchi, C.; Brunelli, C.; Cordero, C.; Rubiolo, P.; Galli, M.; Sironi. A.; J. Chromatogr., A 2005, 1071, 3 .

29. Facchetti, R.; Cadoppi, A.; Rev. Anal. 2005, 16, 63.

30. Ficarra, A.; Fiego, D. P.; Minelli, G.; Antonelli, A.; Food Chem. 2010, $121,809$.

31. Povolo, M.; Pelizzola, V.; Contarini, G.; Eur. J. Lipid. Sci. Technol. 2008, 110,1050

32. Cavagnino, D.; Munari, F.; Pisani, S.; Anais XII Congresso Latino Americano de Cromatografia e Técnicas Relacionadas - Colacro, Florianópolis, Brasil, 2008.

33. Luong, J.; Gras, R.; Jennings, W.; J. Sep. Sci. 2007, 30, 2480.

34. Pierone, D.; $10^{a}$ Edição do Congresso Analitica Latin America, São Paulo, Brasil, 2009.
35. Mastovska, K.; Lehotay, S. J.; J. Chromatogr., A 2003, 1000, 153.

36. Amirav, A.; Tzanani, N.; Wainhaus, S. B.; Dagan, S.; Eur. Mass Spectrom. 1998, 4, 7.

37. Dömötörová, M.; Kirchner, M.; Matisová, E.; Zeeuw, J.; J. Sep. Sci. 2006, 29, 1051

38. Facchetti, R.; Cadoppi, A.; LCGC Europe 2005, 18, 338.

39. http://www.analiticaweb.com.br/newsletter/07/10132.pdf, acessada em Outubro 2010.

40. Ehrmann, E. U.; Dharmasena, H. P.; Carney, K.; Overton, E. B.; J. Chromatogr. Sci. 1996, 34, 533.

41. Jain, V.; Phillips, J. B.; J. Chromatogr. Sci. 1995, 33, 55.

42. Phillips, J. B.; Jain, V.; J. Chromatogr. Sci. 1995, 33, 541

43. Overton, E. B.; Carney, K. R.; Roques, N.; Dharmasena, H. P.; Field Anal. Chem. Tech. 2001, 5, 97.

44. Sloan, K. M.; Mustacich, R. V.; Eckenrode, B. A.; Field Anal. Chem. Tech. 2001, 5, 288.

45. van Deursen, M.; Beens, J.; Cramers, C. A.; J. High Resol. Chromatogr. 1999, 22, 509.

46. van Deursen, M.; Janssen, H. G.; Beens, J.; Rutten, G.; Cramers, C. A.; J. Microcolumn Sep. 2001, 13, 337.

47. Mustacich, R. V.; US pat. 5,782,964 1998.

48. Mustacich, R. V.; US pat. 6,490,852 2002.

49. Mustacich, R. V.; James, F. E.; US pat. 6,682,699 2004.

50. Magni, P.; Zilioli, G.; Facchetti, R.; US pat. 7,228,067 2007.

51. Dallüge, J.; Vreuls, R. J. J.; van Iperen, D. J.; van Rijn, M.; Brinkman, U. A.; J. Sep. Sci. 2002, 25, 608.

52. Cavalli, E. J.; Guinchard, C.; J. Chromatogr. Sci. 1995, 33, 573

53. Hinshaw, J. V.; LCGC 2003, 21, 268.

54. Del Grande, M.; comunicação pessoal (Químico de Aplicação - Sinc do Brasil).

55. Gonçalvez, M.; comunicação pessoal (Químico de Aplicação - Instrulab Instrumentos para Laboratório).

56. Pieroni, D.; comunicação pessoal (Manager for Liquid and Gas Chromatography Products - Nova Analítica Importação e Exportação Ltda).

57. Dagan, S.; Amirav, A.; J. Am. Soc. Mass Spectrom. 1995, 6, 120.

58. Dagan, S.; Amirav, A.; Eur. Mass Spectrom. 1998, 4, 15.

59. Quintella, C. M.; Quim. Nova 1996, 19, 660.

60. American Society for Testing Materials; ASTM D 6584: Determination of Free and Total Glycerin in B-100 Biodiesel Methyl Esters By Gas Chromatography, West Conshohocken, 2008.

61. Comité Européen de Normalisation; EN 14105: Fat and oil derivatives - Fatty Acid Methyl Esters (FAME) - Determination of free and total glycerol and mono-, di-, triglyceride contents, Brussels, 2003. 